\title{
Expression and regulation of the ERK1/2 and p38 MAPK signaling pathways in periodontal tissue remodeling of orthodontic tooth movement
}

\author{
LIPING JIANG and ZHEN TANG \\ Department of Orthodontics, Affiliated Stomatological Hospital of \\ Nanchang University, Nanchang, Jiangxi 330006, P.R. China
}

Received March 11, 2016; Accepted August 3, 2017

DOI: $10.3892 / \mathrm{mmr} .2017 .8021$

\begin{abstract}
The present study aimed to investigate the expression and regulation of extracellular signal-regulated kinase (ERK)1/2 and p38 mitogen-activated protein kinase (MAPK) signaling pathways in periodontal tissue remodeling of orthodontic tooth movement. Sprague Dawley rats with orthodontic tooth movement were generated. After tension stress for 1, 3, 5, 7 and 14 days, the protein and mRNA expression levels of ERK1/2 and p38 in periodontal tissue were determined by western blotting and reverse transcription-quantitative polymerase chain reaction (RT-qPCR), respectively. Primary human periodontal ligament cells (hPDLCs) were separated and characterized. Following exposure to centrifugal force for 1, 2, 6, 8 and $12 \mathrm{~h}$, the protein expression levels of ERK1/2 and p38 MAPK, and the mRNA expression levels of ERK1/2, p38 and osteogenesis associated-genes [including alkaline phosphatase (ALP), osteopontin (OPN), collagen I $(\mathrm{Col} \mathrm{I})$, osteocalcin $(\mathrm{OCN})$ and bone sialoprotein (BSP)] were measured. The protein expression levels of ERK1/2 and p38 MAPK in periodontal tissue and hPDLCs treated with stress were similar to those in the control groups. However, compared with the control, the phosphorylation and mRNA expression levels of the genes encoding ERK1/2 and p38 MAPK in orthodontic periodontal tissue and forced hPDLCs were elevated. These increases reached a peak at 5 days for orthodontic periodontal tissue and at $6 \mathrm{~h}$ for forced hPDLCs. In forced hPDLCs, the mRNA expression levels of ALP, OPN, Col I, OCN and BSP were notably and continuously upregulated in a time-dependent manner. In addition,
\end{abstract}

Correspondence to: Professor Zhen Tang, Department of Orthodontics, Affiliated Stomatological Hospital of Nanchang University, 49 Fuzhou Road, Nanchang, Jiangxi 330006, P.R. China E-mail: tangzhen0426@sina.com

Key words: mitogen-activated protein kinase, extracellular signal-regulated kinase, p38, primary human periodontal ligament cells, periodontal tissue remodeling, orthodontic tooth movement
hPDLCs were treated with the ERK1/2 inhibitor, PD098059, and the p38 MAPK inhibitor, SB203580, and the mRNA expression levels of the osteogenesis associated-genes were then measured using RT-qPCR. Following treatment with the ERK1/2 inhibitor and p38 MAPK inhibitor, the mRNA expression levels of ALP, OPN, Col I, OCN and BSP were significantly downregulated. In conclusion, ERK1/2 and p38 MAPK signaling pathways may be positively and closely associated with periodontal tissue remodeling of orthodontic tooth movement.

\section{Introduction}

Orthodontic tooth movement is achieved via the impact of mechanical force on periodontal tissue, which is a remodeling process accompanying bone regeneration in tension areas and bone resorption in pressure areas $(1,2)$. In bone remodeling, the remodeling of alveolar bone is most important. Periodontal tissue is a periosteum located between alveolar bone and tooth root. It possesses the ability to form bone and serve a key role in bone resorption induced by osteoclasts in the pressure side and bone regeneration due to alveolar bone remodeling and osteoblast generation in the stretching side $(3,4)$. Periodontal ligament cells (PDLCs) are primary cells in periodontal tissue. They direct effector cells for mechanical force and fibroblast-like cells with differentiation potential. Under orthodontic force, PDLCs have an osteoblast phenotype and secrete osteoblast-associated proteins; therefore, PDLCs translate orthodontic force into biochemical signals for the reconstruction of periodontal tissue and tooth movement (3-5). Differentiation of PDLCs into osteoblast-like cells, which participate in bone formation and bone resorption, is a critical process in orthodontic tooth movement. Therefore, the translation of mechanical force into biochemical signals is a biological theoretical basis of modern orthodontic treatment.

Orthodontic force may cause adaptive changes in microenvironments, including the cell matrix, cell membrane, cytoskeleton, nucleoprotein and genome. Consequently, signals are transduced to the cell nucleus, regulating the endonuclear genes and stimulating a series of biological reactions. Various signaling pathways, including the 
mitogen-activated protein kinase (MAPK) pathway are involved in this biological process (6-8). The MAPK pathway is composed of a series of Ser/Thr kinases, including extracellular signal-regulated kinase (ERK)1/2, c-Jun N-terminal kinase (JNK), p38 and ERK5 subfamilies, which after cascade phosphorylation, regulate the activity of certain transcription factors. The MAPK signaling pathway is closely associated with bone resorption of osteoclasts and the bone formation of osteoblasts. In vitro cell studies demonstrated that intermittent mechanical force and $10 \%$ cyclical tension stress activated the ERK1/2 and p38 MAPK signaling pathway in human PDLCs (hPDLCs) $(9,10)$. However, to the best of our knowledge, there are no reports demonstrating the time-dependent expression and mechanism of ERK1/2 and p38 MAPK in orthodontic tooth movement in animals. Therefore, in the present study, a rat model of orthodontic tooth movement and ex vivo hPDLCs exposed to centrifugal force were used to investigate the involvement of ERK1/2 and p38 MAPK signaling pathways in orthodontic tooth movement.

\section{Materials and methods}

Materials and animals. Collagenase I, neutral protease II, Dulbecco's modified Eagle's medium (DMEM), penicillin-streptomycin, fetal bovine serum (FBS), TRIzol and MTT reagent were purchased from Thermo Fisher Scientific, Inc. (Waltham, MA, USA). Hank's buffer, radioimmunoprecipitation (RIPA) buffer and protease inhibitors were purchased from Beijing Solarbio Science \& Technology Co., Ltd. (Beijing, China). Pentobarbital (Purity >99\%; lot no. P3761) was purchased from Sigma-Aldrich; Merck KGaA (Darmstadt, Germany). Diethyl pyrocarbonate (DEPC) was purchased from Sangon Biotech Co., Ltd. (Shanghai, China). The bicinchoninic acid (BCA) assay kit, ERK1/2 inhibitor (PD098059), p38 MAPK inhibitor (SB203580), enhanced chemiluminescent (ECL) plus detection reagent, HRP-labeled goat anti-mouse IgG (A0216), HRP-labeled goat anti-rabbit IgG (A0208), and mouse anti-GAPDH monoclonal antibody (AF0006) were purchased from Beyotime Institute of Biotechnology (Haimen, China). Rabbit anti-ERK1/2 (ab54230), phosphorylated (p)-ERK1/2 (ab201015), p38 MAPK (ab197348) and p-p38 (ab47363) polyclonal antibodies were purchased from Abcam (Cambridge, UK). Orthodontic stainless steel wire was from Innovative Material and Devices, Inc. (Shanghai, China).

A total of 60 patients (35 male and 25 female) were recruited in October 2015. Following obtainment of ethical approval from the Medical Ethics Committee, Affiliated Stomatological Hospital of Nanchang University (Jiangxi, China) and written informed consent, human periodontal tissues were collected from healthy premolar teeth, which were extracted from 11-15 year old patients undergoing orthodontic treatment.

The animal studies were approved by the Institutional Animal Care and Use Committee at the Affiliated Stomatological Hospital of Nanchang University. A total of

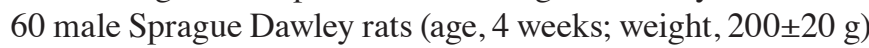
were purchased from Shanghai SLAC Laboratory Animal Co., Ltd. (Shanghai, China) with certificate number SCXK
(Shanghai) 2012-0002. These rats were housed at a temperature of $23 \pm 2^{\circ} \mathrm{C}$ and relative humidity of $\sim 50 \%$, with natural light-dark cycle and free access to water and food. All animal experiments were conducted in compliance with the Guide for the Care and Use of Laboratory Animals of the Affiliated Stomatological Hospital of Nanchang University.

Rats with orthodontic tooth movement. A total of 60 Sprague Dawley rats were randomly assigned into six groups (control, and orthodontic tooth movement for 1, 3, 5, 7 and 14 days; $\mathrm{n}=10$ /group). The right first maxillary molars of the rats were utilized as experimental teeth. Rats were anesthetized with $0.1 \mathrm{ml}$ pentobarbital $(3 \%, \mathrm{w} / \mathrm{v})$, and then retention grooves at lengths of $0.5-1 \mathrm{~mm}$ were made at the near medial surface of the maxillary incisor labial side and right first molar in rats. Spiral springs were ligated with orthodontic stainless steel wire between the maxillary incisor and first molar. Tension force of $\sim 40$ Newtons produced by spiral springs pulled the mesial movement of the first molar. Rats were euthanized at 1 , 3, 5, 7 and 14 days. The first molars and surrounding alveolar bone tissue were collected and stored at $-80^{\circ} \mathrm{C}$ until further analysis.

Ex vivo hPDLCs incubation and characterization. Fresh healthy teeth collected from patients following orthodontic treatment were washed with Hank's buffer containing penicillin-streptomycin to remove bloodstains. One third of the periodontal tissue in tooth roots was scraped using number 12 blades, cut into pieces, and incubated with collagenase I $(3 \%, \mathrm{w} / \mathrm{v})$ and neutral protease II $(4 \%, \mathrm{w} / \mathrm{v})$ for digestion. After $1 \mathrm{~h}$, the mixture was centrifuged for $10 \mathrm{~min}$ at $1,000 \mathrm{x} \mathrm{g}$ at room temperature, and the precipitate was resuspended in DMEM containing $15 \%$ (v/v) FBS to obtain a single cell suspension. The suspension was incubated at $37^{\circ} \mathrm{C}$ and $5 \%$ $\mathrm{CO}_{2}$. The confluence of hPDLCs reached $90 \%$ at day 7 and the cell morphology was observed under a microscope (model TS100, Nikon Corporation, Tokyo, Japan). hPDLCs were long spindle-shaped with a round or oval cell nucleus with a clear nucleolus. Growth was maintained after cell passages.

Treatment of hPDLCs. hPDLCs from passages 2-3 with a good growth status were seeded at a density of $1 \times 10^{4}$ cells/well in 6 -well plates. The plates were placed in a centrifugal bracket at $37^{\circ} \mathrm{C}$ and subjected to a centrifugal force of $80 \mathrm{x}$ g for 1,2 , 6, 8 and 12 h. hPDLCs without treatment served as a control. Subsequently, the protein expression levels of ERK1/2 and p38 MAPK were measured by western blotting. In addition, the mRNA expression levels of ERK1/2, p38 MAPK and osteogenesis-associated genes, including alkaline phosphatase (ALP), osteopontin (OPN), collagen I (Col I), osteocalcin (OCN) and bone sialoprotein (BSP) were measured by reverse transcription-quantitative polymerase chain reaction (RT-qPCR).

hPDLCs were seeded at a density of $1 \times 10^{4}$ cells/well in 6-well plates and treated with $10 \mu \mathrm{M}$ ERK1/2 inhibitor (PD098059) and $10 \mu \mathrm{M}$ p38 MAPK inhibitor (SB203580) at $37^{\circ} \mathrm{C}$ for $24 \mathrm{~h}$. Cells without inhibitor treatment served as the control. Cells with and without treatment were subjected to centrifugal force at $80 \mathrm{x} \mathrm{g}$ and $4^{\circ} \mathrm{C}$ for $6 \mathrm{~h}$. Subsequently, mRNA expression levels of osteogenesis-associated genes, 
Table I. Primer sequences.

\begin{tabular}{ll} 
Gene & \multicolumn{1}{c}{ Primer sequence (5'-3') } \\
\hline ERK1/2 & F: TCAAGCCTTCCAACCTC \\
& R: GCAGCCCACAGACCAAA \\
p38 MAPK & F: AGGGCGATGTGACGTT \\
& R: CTGGCAGGGTGAAGTTGG \\
ALP & F: CTCGTTGACCACCTGGAAGAGCTTCAAACCG \\
OPN & R: GGTCCGTCACGTTGTTCCTGTCAGC \\
& F: CCAAGTAAGTCCAACGAAAG \\
OCN & R: GGTGATGTCCTCGTCTGTA \\
& F: CATGAGAGCCCTCACA \\
BSP & R: AGAGCGACACCCTAGAC \\
& F: AAAACGAAGAAAGCGAAGC \\
Col I & R: TATTCATTGACGCCCGTGTA \\
& F: AGGGCTCCAACGAGATCGAGATCCG \\
GAPDH & R: TACAGGAAGCAGACAGGGCCAACGTCG \\
& F: AGCCACATCGCTCAGACA \\
& R: TGGACTCCACGACGTACT
\end{tabular}

ALP, alkaline phosphatase; BSP, bone sialoprotein; Col I, collagen I; ERK1/2, extracellular signal-regulated kinase 1/2; F, forward; MAPK, mitogen-activated protein kinase; OCN, osteocalcin; OPN, osteopontin; R, reverse.

including ALP, OPN, Col I, OCN and BSP were determined by RT-qPCR.

$R T$ - $q P C R$. In addition to hPDLCs, the mRNA expression levels of ERK1/2 and p38 in homogenized periodontal tissue from rats were determined by RT-qPCR. Total RNA of the periodontal tissue and hPDLCs were extracted using TRIzol reagent and optical density (OD) was measured at $260 \mathrm{~nm}$ (OD260) or $280 \mathrm{~nm}$ (OD280) using a spectrophotometer (Evolution 201/220; Thermo Fisher Scientific, Inc.). The OD260/OD280 ratio ranged between 1.7 and 2.0. RNA was reverse transcribed to cDNA using BeyoRT first strand cDNA synthesis kit (RNase $\mathrm{H}$ minus) (Beyotime Institute of Biotechnology) and a PCR thermal cycler (PTC-200; MJ Research, Inc., Quebec, Canada). Fluorescent quantitation using UltraSYBR mixture (CW0957M, CWBiotech, Beijing, China) was measured on a LightCycler ${ }^{\circledR}$ 96 instrument (Roche Diagnostics, Basel, Switzerland). Primer sequences (Table I) were designed by Sangon Biotech Co., Ltd. A total of $2 \mu \mathrm{l}$ of $1 \mu \mathrm{g}$ RNA, $12.5 \mu \mathrm{l}$ UltraSYBR mixture, $2 \mu \mathrm{l}$ dNTP mixture and $2 \mu 1 \mathrm{MgCl}_{2}$ were mixed and DEPC water $(0.1 \%, \mathrm{v} / \mathrm{v})$ was added to obtain a final volume of $25 \mu \mathrm{l}$. Cycling conditions were as follows: An initial predenaturation step at $95^{\circ} \mathrm{C}$ for $5 \mathrm{~min}$, followed by 40 cycles of denaturation at $95^{\circ} \mathrm{C}$ for $30 \mathrm{sec}$, annealing at $58^{\circ} \mathrm{C}$ for $30 \mathrm{sec}$ and extension at $72^{\circ} \mathrm{C}$ for $30 \mathrm{sec}$. Quantification cycle $(\mathrm{Cq})$ values were recorded and the relative expression level of target genes were calculated using the $2^{-\Delta \Delta \mathrm{Cq}}$ method (11). The average value of three experiments served as the $\mathrm{Cq}$ value of each sample. The $\mathrm{Cq}$ value of the internal control gene subtracted from that of the target gene to generate $\Delta \mathrm{Cq}$, and the average $\Delta \mathrm{Cq}$ of each sample from that of the control equaled $\Delta \Delta \mathrm{Cq}$. The relative expression level of the target gene was calculated using the $2^{-\Delta \Delta \mathrm{Cq}}$ method, and therefore the relative expression level of the control was $2^{0}=1$.
Western blotting. Periodontal tissue of rats or hPDLCs were incubated with $10 \mu \mathrm{g} / \mathrm{ml}$ RIPA buffer and protease inhibitors at $4^{\circ} \mathrm{C}$. The mixture was shaken for $10 \mathrm{~min}$ every $30 \mathrm{sec}$. After $40 \mathrm{~min}$, samples were centrifuged at $4^{\circ} \mathrm{C}$ and 7,000 $\mathrm{x}$ g for $10 \mathrm{~min}$. The supernatant was carefully collected to obtain total protein and the concentration was determined with a BCA assay kit. Proteins $(2 \mu \mathrm{g} / \mu \mathrm{l} ; 10 \mu \mathrm{l})$ were loaded and subjected to $10 \%$ SDS-PAGE, prior to transfer onto a PVDF membrane. The membrane was blocked with $5 \%$ (w/v) non-fat milk at room temperature for $30 \mathrm{~min}$ The membrane was incubated with primary antibodies (anti-GAPDH, anti-ERK1/2, anti-p-ERK1/2, anti-p38 MAPK and anti-p-p38; all 1:1,000) at $4^{\circ} \mathrm{C}$ overnight and subsequently with secondary antibodies (HRP-labeled goat anti-mouse IgG and HRP-labeled goat anti-rabbit $\mathrm{IgG}$; both $1: 1,000$ ) at room temperature for $2 \mathrm{~h}$. ECL plus detection reagent was added to the membrane and proteins were imaged on a ChemiDoc ${ }^{\mathrm{TM}}$ XRS gel imaging system (Bio-Rad Laboratories, Inc., Hercules, CA, USA). Quantity One software (v4.62; Bio-Rad Laboratories, Inc.) was used for densitometric analysis.

Statistical analysis. Experiments were repeated at least three times and data are presented as the mean \pm standard deviation. Statistical analysis was performed using one way analysis of variance followed by a Tukey post-hoc test via SPSS version 17.0 software (SPSS, Inc., Chicago, IL, USA). P<0.05 was considered to indicate a statistically significant difference.

\section{Results}

Protein expression levels of ERK1/2 and p38 MAPK in periodontal tissue of rats after tension force. The protein expression levels of ERK1/2 and p38 MAPK in periodontal 


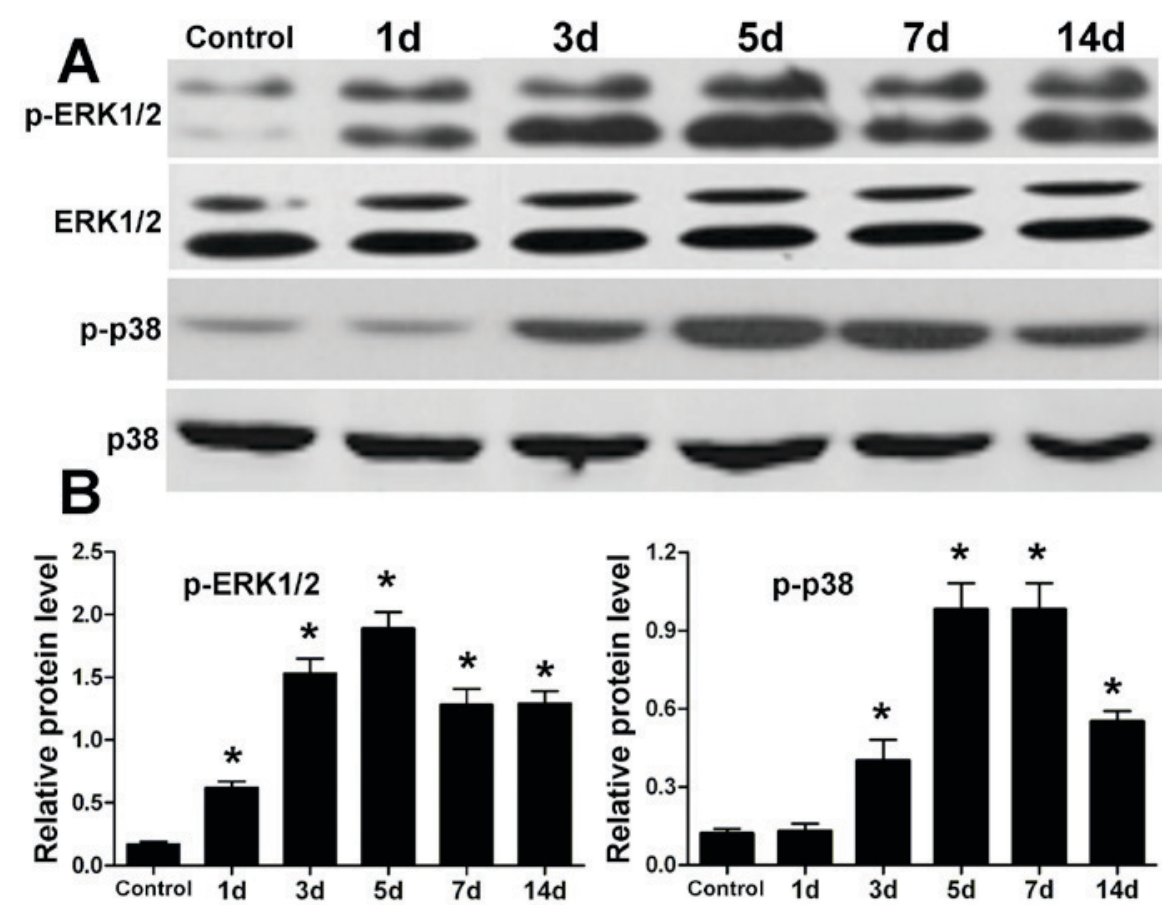

Figure 1. Protein expression levels of ERK1/2 and p38 in periodontal tissue following tension force. (A) Western blotting of ERK1/2, p38 MAPK, p-ERK1/2 and p-p38 MAPK. (B) Semi-quantitative densitometric analysis of p-ERK1/2 and p-p38 after 1, 3, 5, 7 and 14 days of orthodontic tooth movement. ${ }^{*}<<0.05$ vs. the control group. ERK1/2, extracellular signal-regulated kinase 1/2; MAPK, mitogen-activated protein kinase; p-, phosphorylated.
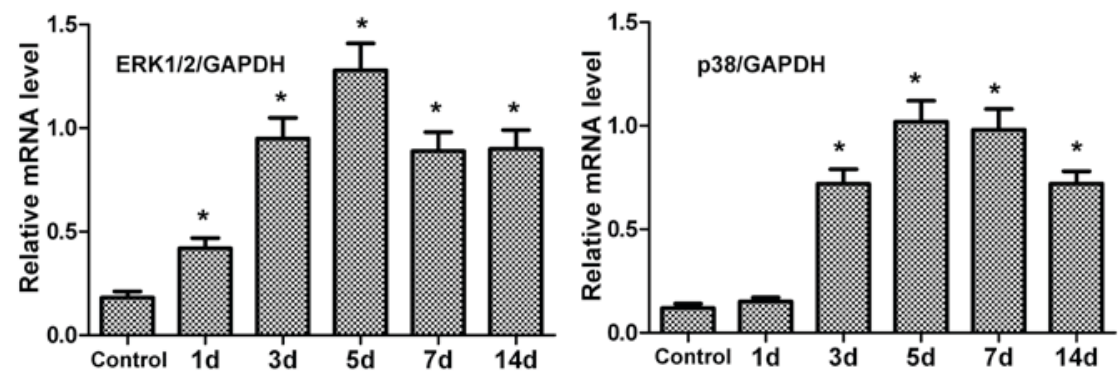

Figure 2. mRNA expression levels of ERK1/2 and p38 at different time points in periodontal tissue after tension force, as determined by reverse transcription-quantitative polymerase chain reaction. ${ }^{*} \mathrm{P}<0.05$ vs. the control group. ERK1/2, extracellular signal-regulated kinase 1/2.

tissue were determined by western blotting (Fig. 1). Compared with the control, the expression levels of ERK1/2 and p38 were similar following orthodontic tooth movement; however, the expression levels of p-ERK1/2 and p-p38 were elevated after tension force. In addition, the increase in expression of p-ERK1/2 and p-p38 reached a peak after 5 days of tooth movement. At 7 and 14 days, expression levels of p-ERK1/2 and p-p38 were reduced to some extent, although they were greater than the control.

mRNA expression levels of ERK1/2 and p38 in periodontal tissue of rats after tension force. The mRNA expression levels of ERK1/2 and p38 in periodontal tissue were measured by RT-qPCR. Following tension force, the mRNA expression levels of ERK1/2 and p38 in periodontal tissue were upregulated compared with the control (Fig. 2). In addition, peak expression was observed at 5 days.

Protein expression levels of ERK1/2 and p38 MAPK in hPDLCs treated with centrifugal force. Protein expression levels of ERK1/2 and p38 MAPK in hPDLCs treated with centrifugal force for various time points were measured by western blotting. There was no difference in the protein levels of ERK1/2 and p38 MAPK in hPDLCs between the control and treatment groups (Fig. 3). However, the expression levels of p-ERK1/2 and p-p38 were elevated in the treatment groups compared with in the control group (Fig. 3). After 1 to $6 \mathrm{~h}$ centrifugal force, there was a gradual increase in the phosphorylation levels of ERK1/2 and p38; however, at $12 \mathrm{~h}$, the phosphorylation of ERK1/2 and p38 MAPK proteins was reduced, although still not to the levels of the control (Fig. 3). These results in hPDLCs were similar to those observed in periodontal tissue.

mRNA expression levels of encoding ERK1/2 and p38 in $h P D L C$ s treated with centrifugal force. The mRNA expression levels of ERK1/2 and p38 in treated hPDLCs were greater than the control (Fig. 4). Under centrifugal force, mRNA expression of these genes gradually increased with time, reaching a peak at $6 \mathrm{~h}$. However, at $12 \mathrm{~h}$, the expression was reduced, although 


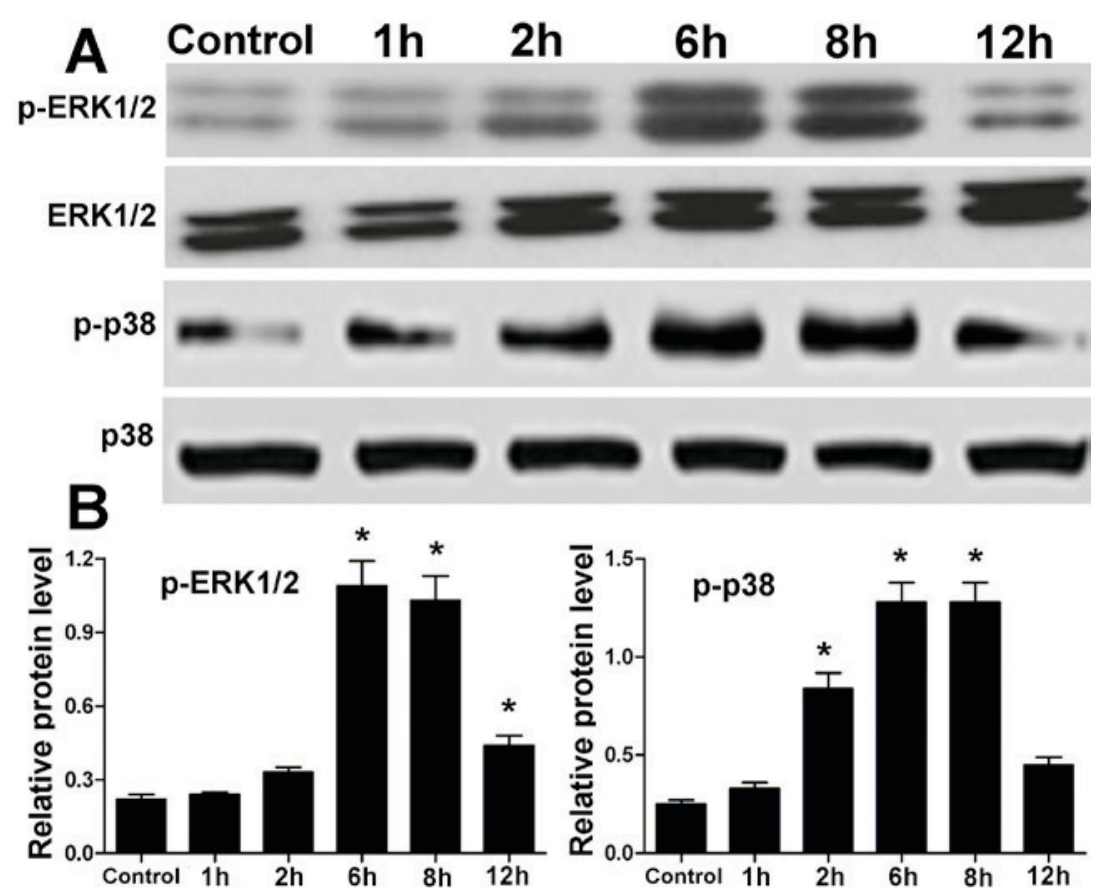

Figure 3. Protein expression levels of ERK1/2 and p38 in hPDLCs treated with centrifugal force. (A) Western blotting of ERK1/2, p38 MAPK, p-ERK1/2 and p-p38 MAPK. (B) Semi-quantitative densitometric analysis of p-ERK1/2 and p-p38 MAPK in the control cells, and in hPDLCs exposed to centrifugal force for various durations. " $\mathrm{P}<0.05$ vs. the control group. ERK1/2, extracellular signal-regulated kinase 1/2; hPDLCs, human periodontal ligament cells; MAPK, mitogen-activated protein kinase; p-, phosphorylated.
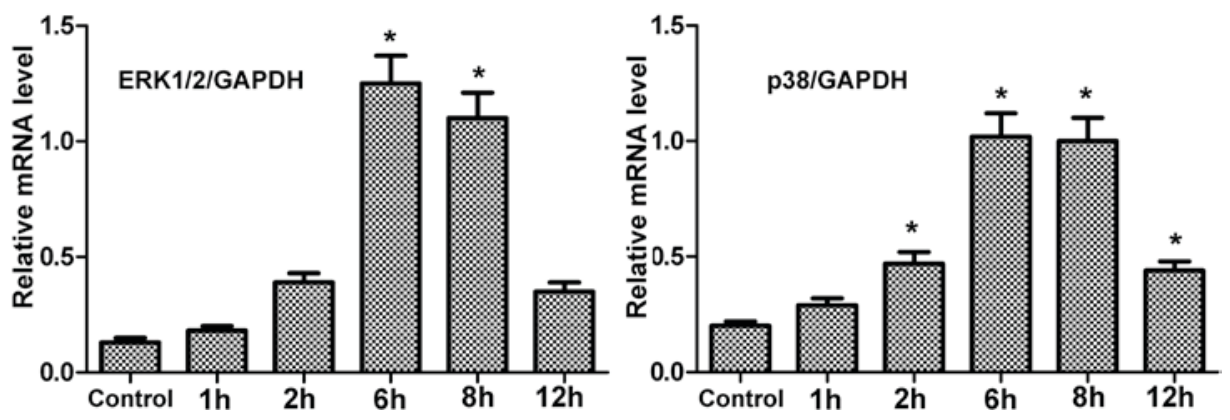

Figure 4. mRNA expression levels of ERK1/2 and p38 in human periodontal ligament cells following exposure to centrifugal force for different time points, as determined by reverse transcription-quantitative polymerase chain reaction. " $\mathrm{P}<0.05$ vs. the control group. ERK1/2, extracellular signal-regulated kinase $1 / 2$.

not to the level of the control. Therefore, the results in hPDLCs concurred with those of periodontal tissue.

Expression of osteogenesis-associated genes in hPDLCs exposed to centrifugal force. Following force treatment, the mRNA expression levels of ALP, OPN, Col I, OCN and BSP were markedly upregulated in hPDLCs compared with in the control group, in a time-dependent manner (Fig. 5). The increase in ALP, OPN, Col I was evident at $1 \mathrm{~h}$ after force treatment, whereas a marked increase in OCN and BSP was observed at $6 \mathrm{~h}$ post treatment.

Expression of osteogenesis-associated genes in hPDLCs following treatment with an ERK1/2 inhibitor and p38 $M A P K$ inhibitor. hPDLCs were treated with an ERK1/2 inhibitor (PD098059) (Fig. 6A) or a p38 MAPK inhibitor (SB203580) (Fig. 6B), and the mRNA expression levels of osteogenesis-associated genes were measured by RT-qPCR. Cells without inhibitor treatment served as the control.

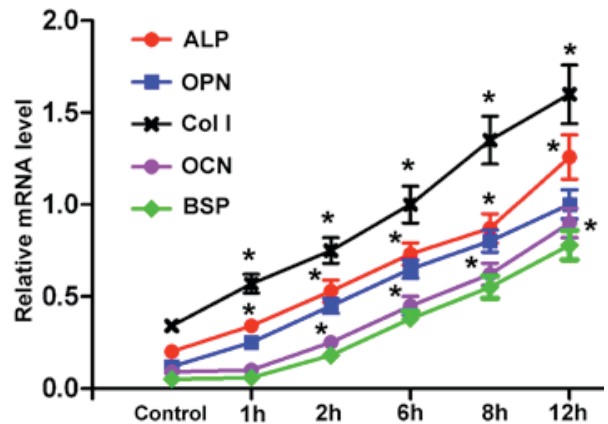

Figure 5. mRNA expression of osteogenesis-associated genes in the control cells or human periodontal ligament cells following exposure to centrifugal force for different time points, as determined by reverse transcription-quantitative polymerase chain reaction. ${ }^{*} \mathrm{P}<0.05$ vs. the control group. ALP, alkaline phosphatase; BSP, bone sialoprotein; Col I, collagen I; OCN, osteocalcin; OPN, osteopontin.

Compared with the control group, the mRNA expression levels of ALP, OPN, Col I, OCN and BSP were significantly 

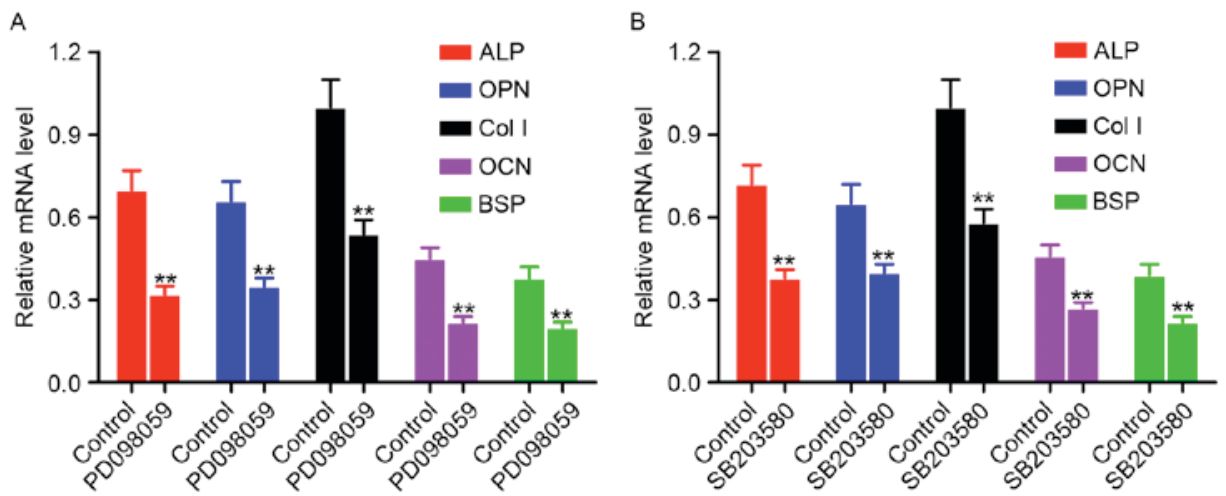

Figure 6. mRNA expression levels of osteogenesis-associated genes in hPDLCs following treatment with (A) extracellular signal-regulated kinase 1/2 inhibitor (PD098059) or (B) p38 mitogen-activated protein kinase inhibitor (SB203580), as determined by reverse transcription-quantitative polymerase chain reaction. ${ }^{* *} \mathrm{P}<0.01$ vs. the control group. ALP, alkaline phosphatase; BSP, bone sialoprotein; Col I, collagen I; hPDLCs, human periodontal ligament cells; OCN, osteocalcin; OPN, osteopontin.

downregulated in the PD098059 or SB203580 treatment groups $(\mathrm{P}<0.01)$.

\section{Discussion}

MAPK is a Ser/Thr protein kinase that is widely expressed in various cells. Through a cascade reaction, the MAPK pathway transduces signals to the cell nucleus to regulate transcription, and therefore influences cell proliferation, apoptosis and differentiation (12). In the MAPK family, ERK1/2, p38 and JNK subfamilies have been extensively investigated. ERK1/2 and p38 are involved in osteogenesis-associated gene expression and bone formation in vivo $(13,14)$. However, the full underlying mechanism of ERK1/2 and p38 pathways in osteogenic differentiation remains contradictory. Li et al (14) revealed that when the ERK1/2 and p38 signaling pathways were inhibited in dental follicle cells, osteogenic differentiation in early, middle and advanced stages was promoted. However, Xiao et al (15) reported that inhibition of ERK1/2 resulted in restrained osteoblast activity and bone formation. Therefore, ERK1/2 may exert positive or negative effects on osteogenic differentiation, which may result from diverse external stimuli, cell types and action time.

Kang et al (16) demonstrated that numerous pathways, including MAPK, were activated in hPDLCs under mechanical pressure. Tsutsumi et al (9) revealed that intermittent mechanical force induced activation of the ERK1/2 and p38 MAPK pathways in hPDLCs. Cyclical tension stress of $10 \%$ promoted the differentiation of hPDLCs via activation of the ERK1/2 signaling pathway and the expression of osteogenic differentiation-associated genes (10). In addition, after continuous stress with 0.25 and 0.5 Newtons in rats, the expression levels of p-ERK1/2 were upregulated (17). It was suggested that in in vitro cell experiments, mechanical force had a positive effect on the ERK1/2 and p38 MAPK signaling pathways. Therefore, to further clarify the role of ERK1/2 and p38 MAPK pathways in orthodontic bone remodeling, the present study used a rat model of orthodontic tooth movement via a spring tension method and a hPDLCs model with centrifugal force. Following tension stress for $1,3,5,7$ and 14 days in vivo or centrifugal force for $1,2,6,8$ and $12 \mathrm{~h}$ ex vivo, the protein and mRNA expression levels of ERK1/2 and p38 in periodontal tissue or hPDLCs were measured by western blotting and RT-qPCR, respectively. The results demonstrated that the mRNA and protein expression levels of p-ERK1/2 and p-p38, and the mRNA expression levels of ERK1/2 and p38, in periodontal tissue and hPDLCs were markedly elevated. Only the mRNA expression levels of ERK1/2 and p38 were increased; total protein levels were not increased. This might result from the increased phosphorylation of ERK1/2 and p38 proteins following activation of the signal pathways. The expression levels of these genes were affected in a time-dependent manner, and the greatest expression was observed at 5 days (in rats) or $6 \mathrm{~h}$ (in hPDLCs). These findings indicated that orthodontic tension stress in rats and hPDLCs exposed to centrifugal force resulted in the phosphorylation of ERK1/2 and p38. Although the transcription of mRNA was initiated at a time that was earlier than the expression time of proteins, the expression of mRNA and protein were continuous and quick processes. This may not always be in a time-dependent manner. Therefore, in the present study, the time-dependent alterations of MAPK protein and mRNA were consistent.

Bone formation involves osteoblast proliferation, extracellular matrix formation and matrix mineralization, and is associated with the expression of numerous genes, including ALP, OPN, Col I, OCN and BSP (18). ALP is primarily involved in initiating matrix mineralization and has a suppressive effect on calcification inhibition. Its expression is an early indicator of osteogenic differentiation and is a precondition for OCN expression and calcium nodule formation (19). As an early-to-mid osteogenic marker, OPN binds to cells or the matrix surface, and promotes the interaction between cells and the extracellular matrix and hydroxyapatite production, thereby facilitating the organization of osteoblast-like-cells, calcium compound deposition and bone formation (20). Col I is a primary scaffold of matrix mineralization and promotes osteoblast adhesion and differentiation (21). As a vitamin K-dependent low molecular weight protein, OCN exhibits a strong affinity for calcium and hydroxyapatite, and is considered an indicator of middle or advanced stage osteogenesis (22). As an extracellular matrix protein, BSP is a marker of osteoblast maturation and is expressed at the advanced stages of bone formation (23). Therefore, the present study measured the mRNA expression levels of osteogenesis-associated 
genes, including ALP, OPN, Col I, OCN and BSP, in hPDLCs following centrifugal force treatment for 1, 2, 6, 8 and $12 \mathrm{~h}$. The expression levels of these genes increased by $6 \mathrm{~h}$. These results suggested that mechanical force positively regulated the expression of osteogenesis-associated genes in hPDLCs. The increase in mRNA expression levels of ALP, OPN, Col I, OCN and BSP in hPDLCs induced by mechanical force was associated with activation of the ERK1/2 and p38 signaling pathways. Expression levels of OCN, BSP, p-ERK1/2 and p-p38 were greatest at $12 \mathrm{~h}$ under stress, and this suggested that the phosphorylation of ERK1/2 and p38 was required for transcription of OCN and BSP. After $6 \mathrm{~h}$, the expression levels of p-ERK1/2 and p-p38 were reduced; however, the expression of osteogenesis-associated genes continued to be upregulated. It was suggested that these five genes may be influenced by other signaling pathways, including bone morphogenetic protein/Smad, Wnt/ $\beta$-catenin, Notch and Hedgehog (24-26).

In addition, Tang et al (27) revealed that flow shear stress on hPDLCs for $2 \mathrm{~h}$ resulted in enhancement of cell viability, ALP activity, Col I mRNA levels, p-ERK1/2, p-p38 MAPK, generation of osteoid-like nodules and rearrangement of filamentous actin. However, following administration of MAPK inhibitors U0126 and SB203580, the alterations induced by flow shear stress were suppressed (24). It was suggested that flow shear stress stimulated osteogenic differentiation of hPDLCs via the ERK1/2 and p38 MAPK signaling pathways. Activation of the ERK1/2 pathway and upregulation of osteogenesis-associated genes may accelerate osteogenesis and calcification of hPDLCs $(8,28)$. Therefore, in the present study, the effects of an ERK1/2 inhibitor and p38 MAPK inhibitor on osteogenesis-associated genes in hPDLCs were investigated. RT-qPCR demonstrated that the ERK1/2 inhibitor and p38 MAPK inhibitor significantly suppressed the mRNA expression levels of ALP, OPN, Col I, OCN and BSP, which suggested that suppression of the ERK1/2 and p38 MAPK pathways may inhibit expression of these osteogenesis-associated genes and bone formation of hPDLCs.

In conclusion, the expression and regulation of ERK1/2 and p38 MAPK signaling pathways were investigated in periodontal tissue following orthodontic tooth movement in vivo and in hPDLCs exposed to centrifugal force ex vivo. The protein expression levels of p-ERK1/2 and p-p38, rather than total ERK1/2 and p38, were elevated in orthodontic periodontal tissue in vivo and hPDLCs ex vivo. The mRNA expression levels of ERK1/2 and p38 in orthodontic periodontal tissue in vivo and hPDLCs ex vivo were additionally upregulated and positively regulated the expression levels of osteogenesis-associated genes, which was further demonstrated using specific inhibitors of ERK1/2 and p38 signaling. ERK1/2 and p38 MAPK pathways were positively and closely associated with periodontal tissue remodeling of orthodontic tooth movement. These findings aid further investigation of the underlying molecular mechanisms in periodontal tissue following orthodontic tooth movement, and the development of drugs that target the ERK1/2 and p38 MAPK signaling pathways.

\section{References}

1. Liu X and Li Z: Research progress of biochemical markers changes of bone turnover in orthodontic tooth movement. J Oral Sci Res 29: 487-489, 2013.
2. Adusumilli S, Yalamanchi L and Yalamanchili PS: Periodontally accelerated osteogenic orthodontics: An interdisciplinary approach for faster orthodontic therapy. J Pharm Bioallied Sci 6 (Suppl 1): S2-S5, 2014.

3. Zhou $\mathrm{H}$ and Cai P: Research progress on periodontal conditions in subjects following orthodontic therapy. Int J Oral Sci 38: 109-111, 2011.

4. Peng $\mathrm{P}$ and Wang W: Biological behavior of periodontal ligament in orthodontic tooth movement. Beijing J Stom 20: 354-357, 2012.

5. Bao X and Hu M: Research progress on mechanisms and regulations of bone resorption in orthodontic tooth movement. Int $\mathrm{J}$ Oral Sci 39: 187-189, 2012.

6. Kim IS, Jeong BC, Kim OS, Kim YJ, Lee SE, Lee KN, Koh JT and Chung HJ: Lactone form 3-hydroxy-3-methylglutaryl-coenzyme a reductase inhibitors (statins) stimulate the osteoblastic differentiation of mouse periodontal ligament cells via the ERK pathway. J Periodontal Res 46: 204-213, 2011.

7. Chen X, Shi J, Ye Q, Cai X and Zhang T: Effect of p38 MAPK signaling pathway on BMP-2-induced osteogenic differentiation of human dental follicle cells. Chin J Cell Bio 35: 816-823, 2013 (In Chinese).

8. Liu C and Sun J: Hydrolyzed tilapia fish collagen induces osteogenic differentiation of human periodontal ligament cells. Biomed Mater 10: 065020, 2015.

9. Tsutsumi T, Kajiya H, Fukawa T, Sasaki M, Nemoto T, Tsuzuki T, Takahashi Y, Fujii S, Maeda H and Okabe K: The potential role of transient receptor potential type A1 as a mechanoreceptor in human periodontal ligament cells. Eur J Oral Sci 121: 538-544, 2013.

10. Li L, Han M, Li S, Wang L and Xu Y: Cyclic tensile stress during physiological occlusal force enhances osteogenic differentiation of human periodontal ligament cells via ERK1/2-Elk1 MAPK pathway. DNA Cell Biol 32: 488-497, 2013.

11. Livak KJ and Schmittgen TD: Analysis of relative gene expression data using real-time quantitative PCR and the 2 (-Delta Delta C(T) method. Methods 25: 402-408, 2001.

12. Gerdts J, Summers DW, Milbrandt J and DiAntonio A: Axon self-destruction: New links among SARM1, MAPKs, and NAD+ metabolism. Neuron 89: 449-460, 2016.

13. Kim HK, Kim MG and Leem KH: Effects of egg yolk-derived peptide on osteogenic gene expression and MAPK activation. Molecules 19: 12909-12924, 2014.

14. Li C, Yang X, He Y, Ye G, Li X, Zhang X, Zhou L and Deng F: Bone morphogenetic protein-9 induces osteogenic differentiation of rat dental follicle stem cells in P38 and ERK1/2 MAPK dependent manner. Int J Med Sci 9: 862-871, 2012.

15. Xiao G, Jiang D, Gopalakrishnan R and Franceschi RT: Fibroblast growth factor 2 induction of the osteocalcin gene requires MAPK activity and phosphorylation of the osteoblast transcription factor, Cbfa1/Runx2. J Biol Chem 277: 36181-36187, 2002.

16. Kang KL, Lee SW, Ahn YS, Kim SH and Kang YG: Bioinformatic analysis of responsive genes in two-dimension and three-dimension cultured human periodontal ligament cells subjected to compressive stress. J Periodontal Res 48: 87-97, 2013.

17. Pavlidis D, Bourauel C, Rahimi A, Götz W and Jäger A: Proliferation and differentiation of periodontal ligament cells following short-term tooth movement in the rat using different regimens of loading. Eur J Orthod 31: 565-571, 2009.

18. An J, Yang H, Zhang Q, Liu C, Zhao J, Zhang L and Chen B: Natural products for treatment of osteoporosis: The effects and mechanisms on promoting osteoblast-mediated bone formation. Life Sci 147: 46-58, 2016.

19. Guo Z, Kang S, Chen D, Wu Q, Wang S, Xie W, Zhu X, Baxter SW, Zhou X, Jurat-Fuentes JL and Zhang Y: MAPK signaling pathway alters expression of midgut ALP and ABCC genes and causes resistance to Bacillus thuringiensis Cry1 Ac toxin in diamondback moth. PLoS Genet 11: e1005124, 2015.

20. Zhang R, Zhang Z, Pan X, Huang X, Huang Z and Zhang G: ATX-LPA axis induces expression of OPN in hepatic cancer cell SMMC7721. Anat Rec (Hoboken) 294: 406-411, 2011.

21. Wang C: The effect of Asiaticoside on the expression of TGF- $\beta$ on thIand Col III in rats after myocardial infarction. J Comm Medi, 2015 (In Chinese).

22. Rocha ÉD, de Brito NJ, Dantas MM, Silva Ade A, Almeida Md and Brandão-Neto J: Effect of zinc supplementation on GH, IGF1, IGFBP3, OCN, and ALP in non-zinc-deficient children. J Am Coll Nutr 34: 290-299, 2015. 
23. Bouet G, Bouleftour W, Juignet L, Linossier MT, Thomas M, Vanden-Bossche A, Aubin JE, Vico L, Marchat D and Malaval L: The impairment of osteogenesis in bone sialoprotein (BSP) knockout calvaria cell cultures is cell density dependent. PLoS One 10: e0117402, 2015.

24. Xu L and Kong Q: Research progress of key signaling pathways in osteoblast differentiation and bone formation regulation. Zhongguo Xiu Fu Chong Jian Wai Ke Za Zhi 28: 1484-1489, 2014 (In Chinese)

25. Yang Z, Ren L, Deng F, Wang Z and Song J: Low-intensity pulsed ultrasound induces osteogenic differentiation of human periodontal ligament cells through activation of bone morphogenetic protein-smad signaling. J Ultrasound Med 33: 865-873, 2014.

26. Matsuzawa M, Sheu TJ, Lee YJ, Chen M, Li TF, Huang CT, Holz JD and Puzas JE: Putative signaling action of amelogenin utilizes the Wnt/beta-catenin pathway. J Periodontal Res 44: 289-296, 2009.
27. Tang M, Peng Z, Mai Z, Chen L, Mao Q, Chen Z, Chen Q, Liu L, Wang Y and Ai H: Fluid shear stress stimulates osteogenic differentiation of human periodontal ligament cells via the extracellular signal-regulated kinase $1 / 2$ and p38 mitogen-activated protein kinase signaling pathways. J Periodontol 85: 1806-1813, 2014.

28. Lee SK, Chung JH, Choi SC, Auh QS, Lee YM, Lee SI and Kim EC: Sodium hydrogen sulfide inhibits nicotine and lipopolysaccharide-induced osteoclastic differentiation and reversed osteoblastic differentiation in human periodontal ligament cells. J Cell Biochem 114: 1183-1193, 2013.

(1) () This work is licensed under a Creative Common Attribution-NonCommercial-NoDerivatives 4.0 International (CC BY-NC-ND 4.0) License. 